\title{
La mort de Corinne: une conversion
}

Sylvie Grignard

D ans le neuvième chapitre de Literary Women: The Great Writers, Ellen Moers banalise l'histoire de Corinne et remet en question son apport féministe chez d'autres écrivaines. Elle écrit: "Corinne stands alone in Mme de Staëls' oeuvre, in its silliness as in its enormous influence upon literary women." (174) En effet, qu'est-ce que Corinne? Sinon un roman d'amour qui, en maintenant la femme dans une dimension onirique, l'empêche d'entrer dans la réalité et de changer son statut politique au sein d'une société patriarchale. Corinne ne sacrifie-t-elle pas jusqu'à mourir d'amour pour un Oswald faible et déloyal? Tout critique féministe, ainsi que l'indique Ellen Moers, se méfie d'un roman d'amour qui aveugle la femme sur la réalité de sa condition en la consolant par des rêves.'

Or de tout temps, depuis Le banquet de Platon et les Héroïdes d'Ovide. pour n'en citer que deux, la dépiction de l'amour n'a été qu'un moyen de codifier les rapports entre indvividus et societé et entre les sexes. ${ }^{2}$ Dans le roman occidental, la femme devient porteuse du destin de l'homme et n'existe par rapport à lui que dans une dimension mythique. ${ }^{3}$ Suivant la tradition romanesque du dix-huitième siècle, elle meurt sacrifiée au devenir du héros. ${ }^{4}$ Or. l'histoire de Corinne flatte l'imaginaire romanesque de son siècle. C'est l'histoire d'une femme qui s'est faite 'ange', comme le dit à maintes reprises Oswald, pour libérer ce dernier de son mal de vivre et mourir abandonnée par lui.

Mais c'est, à mon avis, ranger un peu trop vite Corinne parmi ces romans. Une analyse soignée du développement de l'histoire de Corinne révèle que celle-ci n'aboutit pas à cette conclusion fataliste et stéréotypée des romans du dix-huitième siècle. Corinne ne meurt pas d'amour pour son amant, mais pour d'autres raisons qui, jusqu'à présent, ont échappé à la critique, aveuglée sur les intentions de Germaine de Stäl en tant qu'écrivain.s

Dès le début du roman, la description dichotomique d'Oswald et de Corinne persuade le lecteur de l'incompabilité de leurs caractères et de la mort éminente de Corinne si elle se laisse entraîner à l'aimer. Le narrateur et les propos de Corinne ne cessent d'annoncer sa mort. ${ }^{6}$ Mais page après page, le lecteur 
assiste au spectacle de l'agonie de Corinne sans toutefois en voir la fin. Staël semble ainsi se conformer au modèle littéraire de son époque qui fait savourer au lecteur l'agonie de son hérö̈ne. Cependant, Staël ne sacrifie pas son hérö̈ne pour combler le plaisir sadique de son lecteur. Elle s'amuse à ses dépens en trompant constamment son attente, en déjouant la logique du roman du dixhuitième siècle qui condamne l'héroïne à mourir pour son amant. Corinne, à la différence de Didon, ne meurt pas au moment où elle découvre la trahison de son amant, mais quatre plus tard quand Oswald, marié et père d'une enfant de quatre ans, revient en Italie. L'amour de Corinne pour Oswald n'est donc pas la raison de sa mort. La cause n'en serait-elle pas ailleurs, dans la conclusion au développement même du personnage de Corinne?

Corinne apparait pour la première fois aux yeux du lecteur comme une allégorie de la femme supérieure. L'arrivée de Corinne au Capitole est comparée à celle d'une divinité et indique que son personnage ne représente qu'un mythe. Avec cette description, Staël ne se borne pas, ainsi que Moers le prétend, à décrire "the fantasy of the performing heroine." (174) Staël est consciente que ce fantasme donne l'illusion du pouvoir à la femme dénuée de tout pouvoir politique au sein d'une société patriarcale et qu'en créant cette image boursouflée, inhumaine, d'elle-même qui n'appartient plus à la réalité, la femme s'aliène encore plus de cette société à laquelle elle cherche tant à appartenir. En effet, Staël souligne plusieurs fois l'aliénation de son héroïne. Ses origines sont inconnues et ne lui permettent pas d'avoir une existence personnelle. Corinne n'appartient qu'à son public. Ses talents de danseuse, de musicienne, de peintre, de critique d'art, de chanteuse - et non pas d'écrivain-sont signes de son aliénation. Corinne ne crèe pas, mais interprète. En énumérant la liste des talents de son héroïne et en excluant celui de l'écriture, alors qu'elle même est écrivain, Staël souligne que l'écriture n'est pas admise aux femmes et que leur génie ne peut être qu'amateur. N'ayant pas droit à la parole écrite, elle ne peut créer qu'en interprétant la parole déjà consacrée par l'histoire. Si Corinne fait preuve d'un certain génie créateur, celui-ci n'existe que dans le domaine de l'improvisation où elle doit répondre à l'humeur de son audience sans pouvoir explorer toute la dimension de sa créativité. De plus sa parole, non pas écrite, mais improvisée, se déforme et s'efface avec le temps sans espoir d'être conservée. Son activité créatrice ne peut s'inscrire dans l'histoire.

Staël souligne encore l'aliénation de Corinne en indiquant que son personnage est double. Corinne parle aussi bien l'anglais que l'italien et incarne les caractéristiques opposées de l'Angleterre et de l'Italie. Or, ce manque d'unité interne la fait souffrir. Au Capitole Corinne, malgré toute sa gloire, a une joie mitigée: "on apercevait bien qu'elle était contente d'être admirée; mais un sentiment de timidité se mêlait à sa joie, et semblait demander grace pour son triomphe..." (Corinne 65). Cette timidité, ou tristesse, indique l'insatisfacation interne de Corinne à jouer le rôle de la femme supérieure et de victime destinée au sacrifice. L'intérêt que Corinne porte à l'égard d'Oswald montre son désir d'être unifiée et de mettre fin à son aliénation. Elle commence à s'intéresser à Oswald quand elle remarque qu'il est anglais: "Corinne le remarqua, et bientôt à ses traits, à la couleur de ses cheveux, à son costume, à sa taille élevée, à toutes ses manières enfin, elle le reconnut pour un Anglais" (65). Etant elle-même anglaise par son père, la connaissance d'Oswald représente un lien avec son passé qui l'amènerait à se réhabiliter aux yeux de sa famille et l'ancrerait à nouveau dans 
la réalité historique. Puis, son intérêt s'accroît quand elle remarque qu'Oswald est triste: "Le deuil qu'il portait, et sa physionomie pleine de tristesse la frappèrent" (65). Ignorant alors les différences d 'Oswald, elle identifie sa propre tristesse à celle d'Oswald et cherche en lui une image d'elle-même, projection de son soi sur lequel elle pourra inconsciemment oeuvrer pour tenter d'atteindre son unité intérieure; rétablir le calme chez Oswald devient alors sa raison d'être: "elle devina les pensées qui l'occupaient, et se sentit le besoin de le satisfaire, en parlant du bonheur avec moins d'assurance, en consacrant à la mort quelques vers au milieu d'une fête" (65).

Cette tristesse à laquelle s'identifie Corinne exprime la douleur métaphysique d'Oswald. Après la mort de son père, il ne jouit plus de la vie. S'il ne se suicide pas, il tente par tous les moyens de trouver la mort afin de se libérer du sentiment de culpabilité qui l'oppresse, et de rejoindre son père. Ce dernier. absent, prend aux yeux d'Oswald la dimension d'un dieu qui ne pardonne pas. Oswald est conscient d'avoir fauté en n'obéissant pas aux ordres de son père qui le rappelait à lui. Tiraillé par le remords et le désir de s'en libérer, il essaye de se racheter en contrefaisant sa propre nature afin de correspondre à l'idée que son père se faisait d'un fils. Lui ayant enseigné que le devoir seul est la garantie d'une vie honorable et bonne, Oswald tâche de supprimer la partie sensible de son être en s'accablant sous le poids de diverses responsabilités: "Il espérait trouver dans le strict attachement à tous ses devoirs, et dans le renoncement aux jouissances vives, une garantie contre les peines qui déchirent l'ame..." (28). Cependant, cette attitude n'anihile pas la partie sensible de l'être, mais le divise seulement et détruit l'unité intérieure. La dichotomie de la personnalité d'Oswald est révélée quand il rencontre Corinne au Capitole. Découvrant qu'elle parle aussi bien l'anglais que l'italien, il voit en elle une combinaison des deux éléments séparés de sa propre personnalité - la sensibilité et la raison- qu'il voudrait inconsciemment réunir:

Oswald avait beaucoup de préventions contre

les Italiennes; il les croy ait passionnées, mais

mobiles, mais incapables d'éprouver des affections

profondes et durables.Déjà ce que Corinne avait

dit au Capitole lui avait inspiré toute une autre idée; que

serait-ce donc s'il pouvait à la fois retrouver les souvenirs

de sa patrie, et recevoir par l'imagination une vie nouvelle,

renaître pour l'avenir, sans rompre avec le passé! (69)

Corinne s'aperçoit de la douleur d'Oswald, divisé entre l'avenir et le passé, et s'efforce de l'apaiser. Mais cette douleur ne peut être soulagée que si elle est concrétisée, c'est-à-dire révélée par un contraste. Après avoir improvisé des vers célébrant le bonheur, Corinne compose quelques vers qui sympathisent avec la douleur d'Oswald en la reflétant:

"L'intention de la nature semble trompée, ses

bienfaits inutiles, ses offres repoussées,

et l'expression de la peine, au milieu de

tant de jouissances, étonne, et touche plus

profondément que la douleur chantée dans

les langues du nord, qui semblent inspirées

par elle" (67).

Corinne, en recherchant l'amour et le bonheur en Oswald, c'est-à-dire un lien avec son passé qui lui permettrait de retrouver une existence personnelle, tend à vouloir plaire en spoliant sa propre nature et celle du monde qui l'entoure, un 
monde fait pour rendre tout individu heureux. Elle accroît son aliénation et son désordre intérieur. Oswald, en cherchant continuellement l'unification avec un père qui rejette tout ce que représente Corinne, refuse de rompre avec un passé qui l'empêche de créer son propre devenir et accentue la dichotomie de son entité et de celle de Corinne. Or, plus Oswald est divisé, plus Corinne s'attache à l'unifier; et s'ils ont un but semblable — celui de mettre fin à leur aliénation respective- leurs moyens pour y parvenir sont contradictoires et les enchaînent l'un à l'autre sans espoir de libération.

\section{L'ordre du père}

L'ordre du père est inscrit dans l'histoire de Lord Nelvil et dans celle de Corinne. Ces deux histoires, non seulement apparaissent au centre du roman, mais sont aussi, du point de vue de la narration, centrales puisqu'elles décident de l'avenir des deux protagonistes - l'abandon de Corinne par Oswald-que le narrateur, comme un oiseau de mauvais augure, ne cesse de prédire dans ses descriptions et ses annonces. C'est en effet au moment de ces aveux historiques que les deux protagonistes prennent conscience que ce qui constitue un interdit à leur amour n'est autre que l'ordre du père. Mais quel est cet ordre? Comme le souligne Staël en indiquant qu'Oswald narre son histoire alors que Corinne écrit la sienne, cet ordre à des conséquences différentes pour Oswald et pour Corinne. Oswald est hanté par l'interdit de cet ordre, le père devient un fantôme qui le poursuit (332), alors que Corinne est persécutée par lui.

Dans son récit autobiographique où elle décrit la société anglaise, les femmes et les hommes ont des rôles différents bien déterminés. Les hommes sont libres d'exercer leurs talents dans la sphère publique, mais les femmes, par contre, doivent se confiner à la sphère domestique dans une condition sulbalterne par rapport à l'homme et cacher tout talent qui n'y appartient pas sous peine d'être exclue. Corinne dont le génie dépasse l'espace réservé aux femmes doit s'exiler et renoncer à son état civil.

Dans le cas d'Oswald, cet ordre à d'autres conséquences. Il anihile sa velléité d'indépendance par rapport à cet ordre. En effet, le père d'Oswald est un père manipulateur qui, en semblant aimer sans rien exiger, s'attend à ce que son fils vive sa vie selon des règles de devoir prétablies

Néanmoins il consentit au voyage que je désirais

parce qu'il craignait de rien exiger: il avait une sorte

d'embarras de son autorité paternelle, quand le devoir

ne lui commandait pas d'en faire usage. Il redoutoutait

toujours que cette autorité n'altérât la vérité, la pureté d'affection

qui tient à ce qu'il ya de plus libre et de plus involontaire

dans notre nature, et il avait, avant tout, besoin d'être aimé. (306)

De plus, il inculque à son fils que cet ordre est immuable et absolu et que tenter de le changer n'est que vanité: "Le monde, ce vaste thêâtre, ne change pas d'acteurs; c'est toujours l'homme qui s'y montre en scène; mais l'homme ne se renouvelle point; il se diversifie..." (334). Devant un tel père et un tel enseignement, Oswald ne parvient pas à briser le cordon ombilical qui le lie au père et à vivre ses désirs d'indépendance en assumant sa culpabilité, d'autant plus grande que "l'amour" du père est grand. Cette faiblesse empêche Oswald de s'ancrer dans la réalité. Il apparaît falot et irrésolu. Son manque de courage fait assumer à la femme la responsabilité de ses propres désirs, celle-ci étant fautive quand ils ne correspondent pas aux désirs du père. ${ }^{8}$ 
Le père d'Oswald qui est mort quand commence le roman prend aux yeux d'Oswald la stature d'un dieu absent et tout puissant ainsi qu'il est décrit dans l'Ancien Testament. C'est un dieu aimant, mais légaliste, auquel les hommes doivent sacrifier s'ils veulent être expiés. Dans son amour, Oswald lutte avec lui-même et fait souffrir Corinne. Chacune de ses souffrances est un tribut payé au père. Corinne est identifiée à un Christ, martyre et impuissant. Staël décrit une Corinne qui, pendant la semaine sainte, semble revivre par amour pour Oswald la passion du Christ: “...Corinne vêtue de noir, toute pâle de l'abstinence, et si tremblante dès qu'elle aperçut Oswald, qu'elle fut obligée de s'appuyer sur la balustrade pour avancer: en ce moment le misere commença" (266). Corinne qui a perdu ses droits civils après s'être enfuie d'Angleterre représente une victime désignée et, qui plus est, consentante pour servir d'intermédiaire entre le père et le fils et est donc le pharmakos dans le système sacrificiel décrit par René Girard.'

Toutefois, Staël ne justifie pas cet ordre qui interdit le bonheur des deux protagonistes. Elle insère entre l'histoire de Lord Nelvil et l'histoire de Corinne le livre XIII où se trouve la description majestique du Vésuve qui représente l'ordre triomphant et destructeur d'une nature pervertie, similaire dans ses attributs à celui du père. A plusieurs reprises, Oswald compare l'ordre du père au volcan: "Lord Nelvil vient de mourir, ces lettres étaient flamboyantes; le feu du volcan qui est là devant nous est moins effrayant qu'elles" (332); puis: "cependant cet aspect de l'enfer, tout affreux qu'il est, me cause moins d'effroi que les remords du coeur. Tous les périls peuvent être bravés; mais comment l'objet qui n'est plus pourrait-il nous délivrer des torts que nous nous reprochons envers lui? (338) L'image du père devient celle du volcan qui est un lieu hostile. mystérieux, où l'homme et la nature bienfaisante sont exclus, où l'esprit regénérateur du Nouveau Testament ne pénètre pas: "Jamais le berger ni le pasteur ne conduisent en ce lieu ni leurs brebis ni leurs troupeaux" (304). Des lois inconnues à l'homme, qui peuvent soit le menacer ou le protéger, le régissent:

tout notre être est agité par cette puissance de la

nature, dont les combinaisons sociales nous

avaient distraits longtemps; nous sentons que

les plus grands mystères de ce monde ne consistent

pas tous dans l'homme, et qu'une force indépendante

de lui le menace, ou le protège, selon des lois qu'il

ne peut pénétrer. (293)

L'unité et la puissance du volcan répond au désir de puissance et d'unité de l'homme par la destruction. Tel un dieu, le volcan est encore plus destructeur que l'homme: "la nature n'est plus en ces lieux en relation avec l'homme. Il ne peut plus s'en croire le dominateur; elle échappe à son tyran par la mort" (337). Son unité passe par l'épanchement du feu, du sang et de la destruction comme la lave incendiée qui détruit tout sur son passage pour revenir au moment d'avant la création:

Son éclat est si ardent, que pour la première fois

la terre se réfléchit dans le ciel, et lui donne l'apparence d'un éclair continuel: ce ciel, à son tour, se répète dans la mer, et la nature est embrasée par cette triple image du feu. $(337)^{10}$

La psychologie autodestructrice d'Oswald, créé par l'ordre du père, est un reflet 
des forces destructrices du volcan. La lave qui s'écoule et qui détruit toutes les forces créatrices et bienfaisantes de la nature est l'image des stigmates d'Oswald qui, dans l'indifférence, se laisse souffrir et engendre la souffrance chez Corinne: "mes blessures vont se rouvrir, je le sens; mais en présence de cette nature immuable, faut-il donc avoir tant de peur des souffrances que le temps entraîne avec lui?" (304) Après avoir fait acte d'autopitié en se laissant aller à la souffrance, en narrant son histoire à Corinne, Oswald est devenu apathique et reste prostré. Il ne s'aperçoit plus de la nocivité du volcan et Corinne qui représente symboliquement les forces positives de la vie et, par là même, l'esprit créateur du Nouveau Testament l'en détourne. Elle dit à Oswald: “il me semble voir la nature traitée comme un criminel, et condamnée, comme un être dépravé, à ne plus sentir le souffle bienfaisant de son créateur. Ce n'est sûrement pas ici le séjour des bons, allons-nous-en" (339).

Staël utilise l'image du volcan pour saper plus profondément les racines de l'ordre du père qui apparaissent enracinées dans l'esprit légaliste de l'Ancien Testament. Dans cet ordre, la place de Corinne ne peut être que celle d'une victime sacrifiée, d'un christ martyre et impuissant. Or, ce n'est pas l'image d'une telle Corinne que Staël donne à la fin de son roman. Si Corinne meurt, Staël souligne le caractère libérateur et unificateur de sa mort. L'esprit regénérateur qui souffle à travers le Nouveau Testament et qui est incarné symboliquement par le personnage de Corinne triomphe, par conséquent, de l'ordre légaliste incarné par le père et Oswald.

\section{Corinne: un nouveau Christ}

Le roman aurait dû, soit continuer ainsi qu'il avait commencé, soit se terminer par la mort tragique de Corinne quand elle découvre qu'Oswald aime sa demi-soeur. Toutefois, Staël choisit de lui donner une autre tournure en brisant le cercle vicieux de cette passion. Elle écrit au moment où Corinne est prête à annoncer à Oswald sa venue en Ecosse: "Si elle avait suivi ce mouvement, combien sa destinée et celle d'Oswald eût été différente!" (501)

Le changement de destinée de Corinne n'est dû qu'à un incident banal. Corinne est arrivée en Ecosse pour découvrir pourquoi Oswald , parti rejoindre son régiment depuis plus d'un an, n'a pas donné signe de vie. Or l'évanouissement de Lucile, croyant voir sa soeur dans le jardin, crée une commotion qui l'empêche d'entrer en contact avec Oswald: "Tout le monde courut à son secours. Corinne ne trouva plus le domestique auquel elle voulait parler, et se retira plus avant dans l'allée, afin de ne pas être remarquée" (501). Dès ce moment, Staël introduit un changement dans la psychologie de Corinne.

Celle-ci ne cherche plus à entrer en contact avec Oswald, car les liens avec sa demi-soeur, qu'elle n'avait pas revue depuis sa fuite d'Ecosse, sont renoués. C'est à ce moment que Corinne entre en communion avec Lucile. Le nom donné par Staël à ce personnage est significatif. Lucile signifie lumière. C'est en retrouvant Lucile, la lumière, que Corinne cesse de s'aliéner dans sa passion avec Oswald, qu'elle subit une véritable conversion et qu'elle acquière son unité intérieure. Lucile est le double , l'image inversée de Corinne. Lucile est blonde, pâle, docile, réservée et mystérieuse: l'image de la Madone della Scala. Corinne est brune, enthousiaste, extravertie et créatrice: l'image de la Sibylle peinte par Le Dominiquin. Ces deux images sont celles de la femme créées par l'ordre du père et qu'Oswald n'arrive pas à réunir. Jusqu'à la fin du roman, Oswald sera divisé et hanté par ces deux images. 
De plus, à Lucile est attachée l'image de la lune. Après son évanouissement, elle va sur le tombeau de son père, qui est aussi celui de Corinne, pour prier. Corinne, cachée derrière les arbres l'aperçoit: "elle voyait facilement sa soeur qu'un rayon de la lune éclairait doucement..." (502). Or, la lune est une image chargée de présages qui apparaît fréquemment dans Corinne. Tout au long du roman, alors que Corinne est en présence d'Oswald, la lune n'apparaît jamais totalement. La lumière qu'elle émet est voilee ou contrastée par des forces funestes. Alors que Corinne visite Naples avec Oswald, elle lui dit:

je me disais que ces moments que je passais

avec vous à présent étaient les plus heureux de ma vie: et comme je tournais mes regards vers le ciel pour l'en remercier,je ne sais par quel hasard une superstition de mon enfance s'est ranimée dans mon coeur. La lune que je contemplais s'est couverte d'un nuage et l'aspect de ce nuage était funeste. J'ai toujours trouvé que le ciel avait une impression, tantôt paternelle, tantôt irritée, et je vous le dis, Oswald, ce soir il condamnait notre amour. (289)

Plus tard, sur la colline du cap Misène, après avoir entendu Oswald narrer son histoire, elle décide de faire briller ses talents pour une dernière fois mais la lumière de la lune n'apparaît pas dans tout son éclat: "La lune se levait à l'horizon; mais les derniers rayons du jour fendaient encore sa lumière très pâle" (348). A la fin du roman, alors qu'elle est en train de mourir et qu'Oswald est présent, Corinne lui montre encore cette lune voilée:

Elle voulut lui parler, et n'en eut pas la force.

Elle leva ses regards vers le ciel, et vit la lune

qui se couvrait du même nuage qu'elle avait fait

remarquer à Lord Nelvil quand ils s'arrêtèrent sur

le bord de la mer en allant a Naples. Alors elle le

lui montra de sa main mourante, et son dernier

soupir fit retomber cette main. (586)

L'image de la lune est le symbole d'un nouvel ordre fondé sur la compassion qui ne peut se développer en présence des forces de l'ordre du père, incarnées en Oswald. Par contre, Lucile lui donne essort. En la voyant éclairée distinctement par la lune, Corinne ne peut s'empêcher d'être transportée par la compassion: "elle se sentit tout à coup saisie par un attendrissement purement généreux" (502). En entrant de plus en plus en intimité avec sa demi-soeur, ce sentiment devient plus pur que la passion ressentie pour Oswald. Elle: " répandit des pleurs qu'arrachaient de son coeur des sentiments plus purs encore que l'amour" (503). Un mariage spirituel entre les demi-soeurs naît alors de la compassion de Corinne. Plus tard, Lucile enfante d'une enfant qui ressemble étrangement à Corinne. A Oswald qui ne l'a encore jamais vue, Lucile lui présente sa fille: agée de plus de trois ans, avec autant de timidité qu'une femme coupable en pourrait éprouver. Cette petite ressemblait à Corinne: l'imagination de Lucile avait été fort occupée du souvenir de sa soeur pendant sa grossesse; et Juliette, c'était ainsi qu'elle se nommait, avait les cheveux et les yeux de Corinne" (542). ${ }^{11}$ 
Le "mariage" qui s'effectue au moment où Corinne aperçoit sa demisoeur éclairée par la lune illustre son entrée dans la réalité par rapport à une autre histoire, celle des femmes, qui n'a pas encore été écrite dans un ordre patriarcal dominant. La réunion des deux demi-soeurs au-dessus du tombeau du père est symbolique d'un nouvel ordre, d'une nouvelle histoire qui ne peut exister qu'une fois que l'ordre patriarcal légaliste a disparu. En effet, c'est à ce moment que Corinne abandonne Oswald à Lucile. Dès lors, elle se détache du faux espoir d'appartenir à l'ordre patriarcal en épousant Oswald. Elle trouve son identité par rapport à une alliance avec sa demi-soeur qui est aussi une alliance avec sa bellemère car, en abandonnant Oswald à Lucile, elle fait un pacte avec sa belle-mère qui lui permet de retrouver son état civil. Quand Corinne apprend la date du mariage de Lucile avec Oswald, son être subit une conversion totale qui va l'acheminer lentement vers une autre réalité: "il se fit en elle une révolution subite, tous les intérêts de la vie l'abandonnèrent..." (507). Depuis cet instant jusqu'à la fin du roman, Corinne va mourir à elle-même pour pouvoir renaître à la fin comme le Christ. Elle apprend la sincérité et à entrer dans la réalité supérieure de la vie. Elle s'intéresse aux autres par compassion et ne laisse plus le désir de plaire, la passion de la gloire, remplir son existence: "Elle avait encore en parlant l'intérêt qu'inspire la bienveillance; mais le désir de plaire ne l'animait plus" (526). L'habitude de la retraite la rend consciente de l'aspect néfaste de sa passion pour Oswald et lui donne les forces d'y résister. Quand on lui annonce qu'Oswald est de retour, elle refuse de le voir, car elle ne veut plus le laisser se nourrir de sa douleur et ainsi les faire retomber dans l'engrenage de cette passion qui les aliène et les assujettit. Elle écrit à Oswald et souligne l'ascendant virtuel qu'il a toujours sur elle en le comparant à un dieu: "Les divinités, chez les anciens, n'étaient jamais présentes à la mort; je vous éloignerai de la mienne..." (573).

Elle ne réussit à se maîtriser totalement qu'à la fin, au moment de sa dernière représentation qui lui permet de voir Oswald sans être vue. Avant, Corinne se projetait en Oswald dans l'espoir de faire cesser sa dichotomie et créer sa propre unité. Mais à la fin du roman, elle est devenue consciente qu'Oswald a besoin de voir une dichotomie dans les objets qui l'entourent afin d'apaiser sa mélancolie. Elle tente de se venger de son abandon en utilisant le pouvoir aliénant de l'imagination sur Oswald. Elle met en scène la Douleur qu'elle représente par un contraste entre l'apparence physique de la chanteuse et le contenu de ses paroles: "Il y avait un contraste touchant entre ce visage si paisible et si doux, ce visage où les peines de la vie n'avaient encore laissé aucune trace, et les paroles qu'elle allait prononcer" (581). Elle manipule et contrôle les émotions d'Oswald qui ne peut vivre sincèrement et librement, puisqu'il confond toujours la fiction et la réalité. Ses réactions à la représentation par Corinne de Roméo et Juliette l'indiquaient déjà au début du roman: "Dans l'excès de son trouble, il ne savait pas distinguer si c'était la vérité ou la fiction; et se jetant aux pieds de Corinne, il lui dit en anglais ces paroles de Roméo..." (200). Oswald ne sent pas véritablement et ses sentiments, en passant par l'intermédiaire d'images, font qu'il peut être manipulé facilement. ${ }^{12}$ Toutefois, Staël se refuse à créer une Mme de Merteuil et préfère que son héroïne cherche sa vérité et sa libération, non pas dans une relation de pouvoir, mais dans le contrôle d'elle-même. Elle nous décrit une Corinne qui, à sa dernière représentation en public, s'est à jamais libérée du mécanisme sympathique que lui inspirait la douleur d'Oswald et qui 
la subjuguait:

Elle s'assit, chercha des yeux à découvrir Oswald,

l'aperçut, et, par un mouvement tout-à-fait involontaire,

elle se leva, tendit les bras vers lui, mais

retomba l'instant d'après, en détournant

son visage comme Didon lorsqu'elle rencontre

Enée dans un monde où les passions humaines

ne doivent plus pénétrer. (581)

Comme Didon, Corinne a découvert une autre réalité, celle de la foi, totalement indépendante des fluctuations des valeurs mondaines. Ainsi elle découvre les lois du véritables amour où "le sentiment se plaît surtout à donner ce qui n'est pas dû" (578) et qu'elle explique à sa demi-soeur, son double inversé, avant de mourir. Ce testament d'amour l'unifie et lui ouvre les portes de la vie éternelle et de la liberté où les jugements des hommes ne peuvent pénétrer. Elle dit à Lucile: "Il faut que vous soyez vous et moi tout à la fois..." (578). Par contre, lord Nelvil dont le nom signifie "de la ville" ne découvre pas cette réalité et reste après la mort de Corinne auprès de sa femme et de sa fille, divisé et accablé sous le poids du devoir et du remords: "Enfin l'attachement et le devoir le ramenèrent auprès d'elles.... Lord Nelvil donna l'exemple de la vie domestique la plus régulière et la plus pure" (587).

Le roman de Corinne est la description des étapes d'une conversion dont le lecteur lui-même peut faire l'expérience. Le pronom "je", qui parle après la mort dans la dernière phrase du roman et qui se situe au delà de la moralité humaine en disant à propos d'Oswald: "Je l'ignore, et ne veux, à cet égard, ni le blâmer, ni l'absoudre" (587), n'a pas de référant à l'intérieur du roman. Tout lecteur peut donc se substituer à ce pronom "je". Le roman de Corinne est un acte de foi et par conséquent d'amour. C'est une prière que Staël adresse à l'Europe alors dominée par l' égoïsme extrême de la dictature napoléonienne.

Southwest Missouri State University 


\section{Notes}

${ }^{1}$ Ellen Moers écrit:

If women dwell on love they are doing what is expected of the worst of women, who are said to be stupid, sentimental, hysterical creatures incapable of thinking of anything else. And, by the ladies on my left, the radical feminists, they are berated as traitors to their sex, for love is the snare by which most women are made the slaves of men. (143)

${ }^{2}$ Pierre Fauchery souligne cette tendance dans le roman du dix-huitième siècle: Le sexuel que nous y voyons à l'oeuvre ne se confond pas avec le seul appetitus génésique; car la construction élaborée par ce genre autour du sexe agrège à celui-ci des intérêts de plus en plus complexes et divers, oriente vers lui la plupart des contenus que l'inquiétude contemporaine fait découvrir à l'exploration littéraire du monde. (10)

${ }^{3}$ L'homme "figure habituellement en hôte désinvolte ou un peu gauche, et plutôt comme instrument que comme porteur du destin. C'est sur la femme que le XVIIIème siècle aime à placer ce fardeau et ce sacre" (Fauchery 14). ${ }^{4}$ Robert Fabre décrit cette littérature qui célèbre le désir de mourir par amour:

La littérature - elle ne se limite pas au roman - prodigue les désespoirs d'amour qui font désirer la mort et portent jusqu'au suicide... L'amour décevant ici-bas trouve son accomplissement dans la mort, la passion malheureuse s'y résout enfin dans le repos. (456)

${ }^{5}$ Dans son livre Mme de Staël, Renee Winegarten remarque:

"...Corinne remains one of the greatest myth-making books of all time." (87) De même Madelyn Gutwirth, dans Madame de Staël, Novelist: The Emergence of the Artist as Woman, écrit: "Mme de Staël willed her Corinne to be a myth-making novel..." et ajoute:

The Corinne fiction reflects such a dumb-show of revolt.

For the author herself her novel was a hubristic display ending in a failed ritual of rebellion, its heroine's defeat resulting from the author's need to force upon her the world's feminine ideal, that of self-immolation. (207) Mais Nancy K. Miller indique, dans son livre Subject to Change: Reading Feminist Writing, qu'il y a une tradition d'ecriture féminine du roman dans laquelle les mythes crées par la littérature masculine sont réécrits. L'histoire du roman qui semble dérouler selon les paramètres utilisés dans la narration de fictions masculins subit un déplacement (127). Mon analyse se fait dans cette nouvelle optique afin de localiser le déplacement dans Corinne.

'Voici quelques références à la mort de Corinne: "A ces mots une pâleur mortelle couvrit le visage de Corinne, ses yeux se fermèrent...."(354), "Cette 
belle Corinne...ressemblait à ces fleurs encore fraîches et brillantes, mais qu'un point noir causé par une piqûre mortelle menace d'une fin prochaine"(355), "Que signifie donc cette palpitation douloureuse qui soulève mon sein? Ah! mon ami, je ne la redouterais pas, si elle ne m'annonçait que la mort" (401).

${ }^{7}$ Cette sociète anglaise représente l'idéal de toute une élite aristocratique au moment de la révolution française, l'idéal d'une monarchie constitutionelle auquel Staël souscrivait elle-même tout en émettant quelques réticences devant la façon dont les femmes sont écartées de ce pouvoir (voir Oeuvres posthumes de Madame la Baronne de Staël-Holstein 495 - 510).

${ }^{8}$ En effet, l'amour qu'il porte envers Corinne est une répétition de l'amour qu'il avait ressenti envers Madame d'Arbigny et qu'il s'est imaginé être la cause de la mort de son père. Madame d'Arbigny et Corinne sont associées dans l'inconscient d'Oswald. Mais cette fois-ci, en triomphant de son amour pour Corinne, c'est-à-dire de lui-même, il espère obtenir le pardon du père. ${ }^{9}$ René Girard montre dans son livre, La violence et le sacré, que les êtres qui vivent en marge d'une société - le pharmakos — et dont les droits et devoirs sont à peu près inexistants sont sacrifiés aux intérêts de cette même société (28).

${ }^{10}$ Cette image destructrice du volcan représente la Terreur. Les abstractions sur lesquelles se fondaient le système égalitaire de la Terreur tentaient d'effacer l'histoire. Dans les Oeuvres posthumes, Staël écrit: "Les dogmes politiques, si ce nom peut convenir à de tels égarements, régnaient alors, et non les hommes. On voulait quelque chose d'abstrait dans l'autorité, pour que tout le monde fût censé y avoir pris part" (386). Or, elle compare cette destruction de l'histoire pour l'amour de l'égalité à l'activité d'un volcan: "Les querelles religieuses ont provoqué la révolution d'Angleterre; l'amour de l'égalité, volcan souterrain de la France, agissait aussi sur la secte des puritains..."(380). Cette image du volcan est aussi liée au personnage d'Oswald qui tente de faire le vide en lui-même pour se libérer de l'image du père.

"Le prénom de Juliette est significatif. En effet dans Roméo et Juliette , c'est l'héroïne qui survit alors que le héros meurt, divisé entre l'amour et sa fidélité au clan.

${ }^{12}$ Ainsi que Rousseau dans La lettre d'Alembert et Entretien, Staël va à l'encontre des théories esthétiques sur le roman formulées au dix-huitième siècle. Comme l'explique Vivienne Mylne, dans The Eighteenth-Century Novel: Techniques of Illusion, les auteurs du dix-huitième siècle utilisaient tous les moyens pour créer l'illusion de la réalité dans leurs oeuvres afin d'émouvoir et d'éduquer le lecteur. Or comme l'indique Staël, le lecteur risque de confondre réalité et fiction et, en faisant l'expérience de la vie par images interposées, de devenir insensible à la souffrance humaine. 


\section{Ouvrages cités}

Fabre, Robert. La mort au siècle des lumières. Lyon: Presses universitaires de Lyon, 1978.

Fauchery, Pierre. La destinée féminine dans le roman européen du dixhuitième siècle. Paris: Armand Colin, 1972.

Girard, René. La violence et le sacré. Paris: Bernard Grasset, 1972. Gutwirth, Madelyn. Madame de Staël, Novelist: The Emergence of the Artist as Woman. Urbana: University of Illinois Press, 1978.

Miller, Nancy K. Subject to Change: Reading Feminist Writing. New York: Columbia University Press, 1988.

Moers, Ellen. Literary Women: The Great Writers. New York: Doubleday, 1986.

Mylne, Vivienne. The Eighteenth-Century Novel: Techniques of Illusion.

Manchester: UP, 1965.

Oeuvres posthumes de Madame la Baronne de Staël-Holstein.

Précédées d'une notce sur son caractère et ses écrits. Vol. 3. Paris:

Firmin Didot Frères, 1886.

Staël, Madame de. Corinne ou l'Italie. Préface de Simone Balayé. Paris:

Gallimard, 1985.

Winegarten, Renée. Mme de Staël. Learing Spa: Berg Publishers Ltd, 1985. 